\title{
Stability Analysis and Experimental Research on Ultrasonic Cutting of Wave-absorbing Honeycomb Material With Disc Cutter
}

\section{Zongfu Guo ( $\sim$ guozongfu816@163.com )}

Hangzhou Dianzi University

\section{Xin Liu}

Hangzhou Dianzi University

\section{Sufang Yao}

Hangzhou Dianzi University

\section{Baohua Yu}

Hangzhou Dianzi University

Xiaoping Hu

Hangzhou Dianzi University

\section{Hongxian Ye}

Hangzhou Dianzi University

\section{Research Article}

Keywords: Cutting Stability Analysis, Ultrasonic Cutting, Wave-absorbing Honeycomb Material, Disc Cutter

Posted Date: August 26th, 2021

DOl: https://doi.org/10.21203/rs.3.rs-830212/v1

License: (9) This work is licensed under a Creative Commons Attribution 4.0 International License. Read Full License

Version of Record: A version of this preprint was published at The International Journal of Advanced Manufacturing Technology on January 31st, 2022. See the published version at https://doi.org/10.1007/s00170-022-08776-2. 


\title{
Stability Analysis and Experimental Research on Ultrasonic Cutting of
}

\section{Wave-absorbing Honeycomb Material with Disc Cutter}

\author{
Zongfu Guo ${ }^{1}$, Xin Liu ${ }^{1}$, Sufang Yao ${ }^{1}$, Baohua Yu${ }^{1}$, Xiaoping $\mathrm{Hu}^{1}$, Hongxian $\mathrm{Ye}^{1}$
}

\begin{abstract}
:
To address the problem of the poor stability of ultrasonic machining of wave-absorbing honeycomb materials, this paper takes ultrasonic cutting of wave-absorbing honeycomb materials with a disc cutter as the research object and establishes a multi-degree-of-freedom mathematical model of the cutting system based on the relative positions of the tool and the honeycomb material and the motion characteristics of the tool. On this basis, modal analysis of the disc tool and the honeycomb cellular element wall plate is carried out to draw the Lobe diagram of ultrasonic cutting stability, the process experimental parameters are determined according to the solved stability Lobe diagram, and machining stability verification experiments are carried out. The experimental results show that the machining parameters in the stable region of the Lobe diagram result in a neat and clean surface, less fibre pullout, a complete outer substrate, and less tool wear than those in the critical and unstable regions, thus verifying the correctness of the theoretical model and the stability Lobe diagram.
\end{abstract}

Key words: Cutting Stability Analysis, Ultrasonic Cutting, Wave-absorbing Honeycomb Material, Disc Cutter

Wave-absorbing honeycomb is a structural wave-absorbing material developed based on an aramid honeycomb ${ }^{[1]}$, which is based on a hexagonal aramid honeycomb and impregnated with a resin mixed with an acetylene carbon black honeycomb absorbing substance on honeycomb walls. Due to the excellent properties of low density, light weight, heat and corrosion resistance, and high impact resistance ${ }^{[1]}$,wave-absorbing honeycombs are widely used in military, aerospace, and other fields with special requirements ${ }^{[3]}$.

In the cutting processing of wave-absorbing honeycomb materials, the local cutting area is similar to thin-walled plate processing. If the process parameters are not selected properly, the machining state is prone to destabilization and chattering, which leads to fibre pull-out, outer substrate deformation, and coating peeling on the machined surface ${ }^{[3]}$, which seriously affects the surface quality of the workpiece after moulding. To improve the stability of the machining process of thin-walled parts, Bravo ${ }^{[5]}$ assumed thin-walled parts and tools as elastomers, analysed the three-dimensional stability leaflet diagrams of different machining stages by finite elements, and compared the cutting conditions by finite elements, and compared the cutting conditions with

Zongfu Guo guozongfu816@163.com

1 School of Mechanical Engineering, Hangzhou Dianzi University, Hangzhou 310018, China the critical conditions in the leaflet diagrams to derive the optimal machining conditions for low stiffness parts. Thevenot ${ }^{[6]}$ after considering the vibration generated in the milling of thin-walled parts based on the assumption that the thin-walled part is an elastomer and the tool is a rigid body, established a three-dimensional stability Lobe diagram using modal analysis and finite element simulation. It was shown how to use the stability Lobe diagram to determine the changes in the dynamic characteristics of the thin-walled workpiece. Zhang ${ }^{[7]}$ used the matrix ingestion method to predict the dynamic modalities of the workpiece during high-speed milling on the basis of the finite element analysis model and proposed that the material during the removal process dynamic modal changes have an important effect on the whole cutting system. Mancisidor ${ }^{[8]}$ et al. proposed a method for calculating the dynamic behaviour based on the fixed boundary of the tool to obtain chatter-free machining conditions with a dynamic transfer function at the tool tip and performed experimental calculations of the frequency response function considering various combinations of tool and machine tool machining parameters. Xuewei Zhang ${ }^{[9]}$ et al. established a three-dimensional milling dynamics model for thin-walled materials with low stiffness, simulated and verified the critical axial chatter depth of cut versus tool speed using the fully discrete analytical method, and plotted the stability Lobe diagram. Zhu Hui ${ }^{[10]}$ et al. investigated the vibration stability of thin-walled parts in 
longitudinal torsional ultrasonic vibration machining by establishing a two-dimensional milling model of a double-flexible body and studied the effect of different cutting parameters on the chattering stability in depth by finite element simulation. Based on the above research, it can be found that establishing a mathematical model of stability machining and drawing Lobe diagrams of machining process stability provide a theoretical basis for the selection of process parameters to carry out high-quality machining.

In this paper, we investigate the stability processing of wave-absorbing honeycomb materials by ultrasonic cutting technology with disc knives. First, the theoretical analysis of ultrasonic cutting of wave-absorbing honeycomb materials with a disc cutter is carried out, including establishing a multi-degree-of-freedom stability mathematical model of the cutting system and the modal analysis of the disc cutter and the cellular wall plate using finite element software to obtain the dynamic parameters of the tool and the cellular wall plate. Then, the Lobe diagram of the disc cutter machining process stability is drawn. Second, ultrasonic cutting experiments of the disc cutter are carried out, and the correctness of the multi-degree-of-freedom stability mathematical model is verified by examining the surface morphology of the workpiece formed by cutting under each process parameter and the wear condition of the disc cutter.

\section{Ultrasonic cutting of wave-absorbing cellular materials by disc cutter Theoretical analysis}

1.1 Development of a multi-degree-of-freedom stability model for ultrasonic cutting systems

An ultrasonic cutting system is a cutting system with the rotary motion of the disc cutter itself and high-frequency ultrasonic vibration as the main forms of motion. According to the relative position of the tool, the honeycomb material and the motion characteristics of the tool, a three-dimensional coordinate system is established, as shown in Figure 1b). The depth of cut $a_{p}$ and the cutting width $a_{e}$ are set after setting the depth and width of the cut. The honeycomb is cut in the XZ plane with the feed of the tool in the $\mathrm{Z}$ direction of the coordinate axis. The rotational speed of the disc tool is $n$, the feed speed is $V_{f}$ and the motion displacement is $S^{\prime}$.

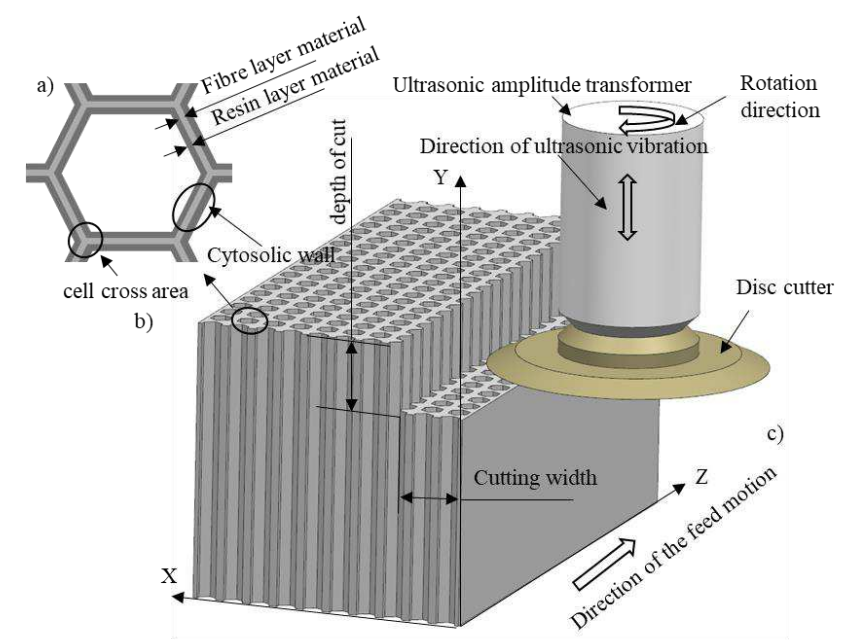

Figure 1 a) Schematic diagram of cellular material cytosolic structure and b) schematic diagram of ultrasonic cutting of honeycomb material by disc cutter

When an ultrasonic wave with amplitude $A$ and frequency $f$ is applied to the tool, the motion of the circular knife relative to the cellular wall plate in the X-direction is only the sub-motion of the rotational motion of the tool, and the equation is

$$
\begin{aligned}
& =R \cdot(1 \\
& \left.-\cos \frac{2 \pi n}{60} t\right)
\end{aligned}
$$

$=\frac{2 \pi n R}{60}$

$\sin \frac{2 \pi n}{60} t$

$$
=\left(\frac{2 \pi n}{60}\right)^{2} R \cdot \cos \frac{2 \pi n}{60} t
$$

In the Y-direction, there is only ultrasonic vibration of the tool, and the equation of motion is

$$
\begin{aligned}
& =A \\
& \cdot \sin (2 \pi f t) \\
& =2 \pi f A \\
& \cdot \cos (2 \pi f t) \\
& \quad a_{y}{ }^{\prime}=-(2 \pi f)^{2} A \cdot \sin (2 \pi f t)
\end{aligned}
$$

In the Z-direction, the motion of the tool includes the sub-motion of feed motion and rotary motion, and the equation of motion is

$=R \cdot \sin \frac{2 \pi n}{60} t$ 


$$
\begin{gathered}
V_{z}{ }^{\prime}=V_{f}+\frac{2 \pi n R}{60} \cdot \cos \frac{2 \pi n}{60} t \\
a_{z}{ }^{\prime}=-\left(\frac{2 \pi n}{60}\right)^{2} R \cdot \sin \frac{2 \pi n}{60} t
\end{gathered}
$$

According to equation (1.4), the ultrasonic vibration displacement of the disc cutter in the $\mathrm{Y}$ direction is very small and almost negligible compared to the rotation and feed motion in the other two directions.

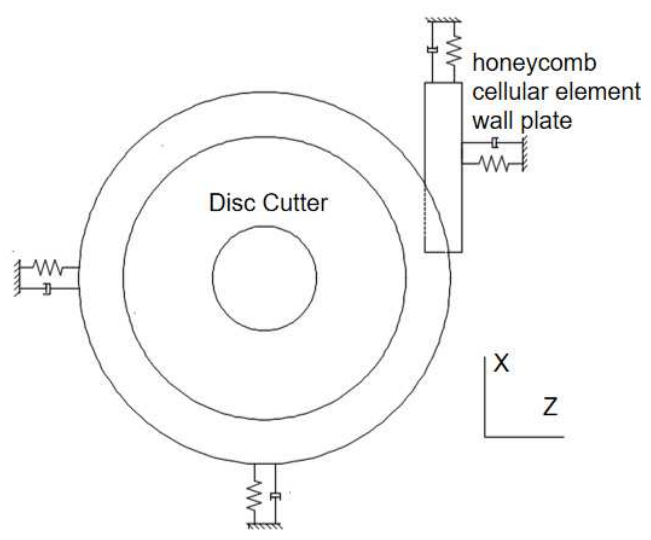

Figure 2 Simplified model of ultrasonic cutting system

This ultrasonic cutting system can be simplified to a two-degree-of-freedom cutting system, as shown in Figure 2, so that the dynamics equation of this ultrasonic cutting system can be obtained as

$$
\left[M_{k^{\prime}}\right]\left\{y_{k^{\prime}}^{\prime \prime}(t)\right\}+\left[C_{k^{\prime}}\right]\left\{y_{k^{\prime}}^{\prime}(t)\right\}+\left[k_{k^{\prime}}\right]\left\{y_{k^{\prime}}(t)\right\}=\left\{F_{k^{\prime}}(t)\right\}
$$

where $\left[M_{k^{\prime}}\right],\left[C_{k^{\prime}}\right]$ and $\left[k_{k^{\prime}}\right]$ are the $2 \times 2$ order mass, damping and stiffness matrices of the disc cutter-cellular element wall plate system, and $\left\{y_{k^{\prime}}^{\prime \prime}(t)\right\},\left\{y_{k^{\prime}}^{\prime}(t)\right\}$ and $\left\{y_{k^{\prime}}(t)\right\}$ and $\left\{F_{k^{\prime}}(t)\right\}$ are the acceleration, velocity, displacement, and cutting force vectors.

When the tool is ultrasonically cut, the cutting force gradually increases with increasing cutting thickness, and the tool vibrates when cutting the honeycomb cellular element wall plate. Thus, the structural mode of the whole cutting system is self-excited and produces unstable chattering. The cutting system is excited by the cutting force in the $\mathrm{X}$-direction and the $\mathrm{Z}$-direction to produce dynamic displacement, and the dynamic displacement of the cutting thickness caused by the vibration between the tool and the honeycomb cellular element wall plate is

$$
s_{k}=x_{k} \cdot \sin \phi \cdot \sin \zeta+z_{k} \cdot \cos \phi \cdot \sin \zeta
$$

where $\phi$ is the radial contact angle of the disc cutter when the tool spindle speed is $\mathrm{n}, \phi=n \pi t / 30 ; \zeta$ is the axial immersion angle, where $\zeta=\pi / 2 ; x_{k}$ 目 $z_{k}$ is the dynamic displacement of the cutting edge.

With the rotation of the tool spindle, the radial contact angle of the tool changes with time, causing the chip thickness to change with time. At this time, the chip thickness includes the static chip thickness caused by the rigid body motion of the tool and the dynamic chip thickness caused by the vibration of two adjacent cycles of the cutting edge, which can be expressed as

$$
\delta_{k}(\phi)=\left[c_{z} \cdot \sin \phi+\left(s_{k-1}-s_{k}\right)\right] \cdot g_{k}(\phi)
$$

where $c_{z}$ is the knife edge feed. $s_{k-1}-s_{k}$ is the difference between the dynamic displacements of the disc cutter in two adjacent cycles. $g_{k}(\phi)$ is the unit step function used to determine whether the cutting edge is making a cut and takes the value of the radial contact angle $\phi$ as the angle of entry $\phi_{\text {in }}$ and cut-out angle $\phi_{\text {out }}$ between, expressed as

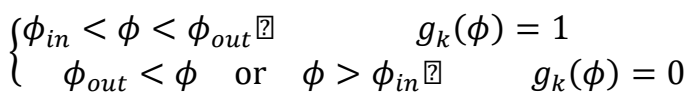

Since the static part of the cutting thickness has no influence on the stability of the system, only the dynamic part of the chip thickness is obtained.

$$
\delta_{k}(\phi)=\left[\Delta x_{k} \cdot \sin \phi+\Delta z_{k} \cdot \cos \phi\right] \cdot g_{k}(\phi)
$$

where $\Delta x_{k}=x_{k}(t)-x_{k}(t-T), \Delta z_{k}=z_{k}(t)-z_{k}(t-T)$ in which $\mathrm{t}$ is the current time and $\mathrm{T}$ is the tool rotation period. $\left(x_{k}(t), z_{k}(t)\right)$ and $\left(x_{k}(t-T), z_{k}(t-T)\right)$ denote the relative dynamic displacement between the Cytomel wall plate and the tool in adjacent cycles, respectively.

Radial and tangential cutting forces on unit arc length $F_{r}, F_{t}$ with $a_{p}$ (axial depth of cut), the $\delta_{k}(\phi)$. The following relationship exists:

$$
\left\{\begin{array}{l}
F_{r}=K_{r} a_{p} \cdot \delta_{k}(\phi) \\
F_{t}=K_{t} a_{p} \cdot \delta_{k}(\phi)
\end{array}\right.
$$

where $K_{r}$ and $K_{t}$ are the radial and tangential cutting force coefficients, respectively. The radial and tangential cutting forces are decomposed to obtain the $\mathrm{X}$ - and Z-directional cutting forces, which are expressed in the matrix as

$$
\left[\begin{array}{l}
F^{X} \\
F^{Z}
\end{array}\right]=\left[\begin{array}{cc}
-\sin \phi & -\cos \phi \\
-\cos \phi & \sin \phi
\end{array}\right]\left[\begin{array}{l}
F_{r} \\
F_{t}
\end{array}\right]
$$

Substituting (1.14) and (1.15) into (1.16) yields the relationship between the cutting force and displacement as $\left[\begin{array}{l}F^{X} \\ F^{Z}\end{array}\right]=$

$$
a_{p}[N]\left[\begin{array}{l}
\Delta x_{k} \\
\Delta z_{k}
\end{array}\right]
$$

where $[N]$ denotes the time-varying dynamic ultrasonic cutting force coefficient, which is a $2 \times 2$ order matrix, and the individual elements of the matrix are

$$
[N]=\left[\begin{array}{ll}
n_{x x} & n_{x z} \\
n_{z x} & n_{z z}
\end{array}\right]=
$$




$$
g_{k}(\phi)\left[\begin{array}{cc}
-\sin \phi & -\cos \phi \\
-\cos \phi & \sin \phi
\end{array}\right]\left[\begin{array}{l}
K_{r} \\
K_{t}
\end{array}\right]\left[\begin{array}{ll}
\sin \phi & \cos \phi
\end{array}\right]
$$

Eq. (1.8) is expressed in the time domain in the form of a matrix as

$$
\left\{F^{k^{\prime}}(t)\right\}=a_{p}[N(t)]\{\Delta(t)\}
$$

where the matrix $\left\{F^{k^{\prime}}(t)\right\}$ represents the dynamic cutting force in the $\mathrm{X}$ and $\mathrm{Z}$ directions; the disc tool then rotates clockwise when the tool enters the cutting state.

$$
[N(t)]=\left[N_{0}\right]=\frac{1}{4}\left[\begin{array}{ll}
\left(-K_{r} \sin 2 \phi-2 K_{r} \phi+K_{t} \cos 2 \phi\right) /_{\phi_{\text {in }}}^{\phi_{\text {out }}} & \left(K_{r} \cos 2 \phi-2 K_{t} \phi+K_{t} \sin 2 \phi\right) /_{\phi_{\text {in }}}^{\phi_{\text {out }}} \\
\left(-K_{r} \cos 2 \phi+2 K_{t} \phi-K_{t} \sin 2 \phi\right) /{ }_{\phi_{\text {in }}}^{\phi_{\text {out }}} & \left.\left(K_{r} \sin 2 \phi-2 K_{r} \phi-K_{t} \cos 2 \phi\right)\right|_{\phi_{\text {in }}} ^{\phi_{\text {out }}}
\end{array}\right]
$$

we have

The matrix $\{\Delta(t)\}$ represents the dynamic displacements in the $\mathrm{X}$ and $\mathrm{Z}$ directions and is expressed in terms of the transfer function matrix of the tool-cylinder wall plate as

$$
=\left(1-e^{-i \omega_{C^{\prime}} T}\right)[\mathrm{H}(s)]\left\{F^{k^{\prime}}(t)\right\}
$$

where $\omega_{C^{\prime}}$ is the chattering frequency; the transfer function matrix $[\mathrm{H}(s)]$ is the transfer function matrix of the disc cutter $\left[H_{C^{\prime}}(s)\right]$, and the transfer function matrix of the cellular wall plate $\left[H_{W}(s)\right]$ is the sum of

$$
\begin{gathered}
{[\mathrm{H}(s)]=} \\
{\left[\begin{array}{ll}
H_{C^{\prime} x x}(s)+H_{W x x}(s) & H_{C^{\prime} x z}(s)+H_{W x z}(s) \\
H_{C^{\prime} z x}(s)+H_{W z x}(s) & H_{C^{\prime} z z}(s)+H_{W z z}(s)
\end{array}\right]}
\end{gathered}
$$

The chattering stability of the system is determined by the characteristic equation of the two-dimensional dynamic ultrasonic cutting system and is given by the following equation.

$$
\operatorname{det}\{[I]+\Lambda \cdot[\Phi]\}=0
$$

where $[I]$ is the unit matrix; $[\Phi]=\left[N_{0}\right][\mathrm{H}(s)]$ is the directional transfer function matrix; the complex eigenvalue $\Lambda=-a_{p}\left(1-e^{-i \omega_{C^{\prime}} T}\right)$, if the cutting force coefficient is given $K_{r}$, the $K_{t}$ the value of, solves for the complex eigenvalue as:

$$
a_{2} \Lambda^{2}+a_{1} \Lambda+1=0
$$

According to Euler's formula, we have $e^{-i \omega_{C^{\prime}} T}=$ $\cos \omega_{C^{\prime}} T-i \sin \omega_{C^{\prime}} T$ and substitute into $\Lambda$.

$\Lambda=-a_{p}\left(1-\cos \omega_{C^{\prime}} T+i \sin \omega_{C^{\prime}} T\right)=\Lambda_{R}+i \Lambda_{I}$

to obtain the critical depth of cut $a_{p}$ of the solution formula for the depth of cut with $\omega_{C^{\prime}}$ and the expression is:

$$
\begin{aligned}
a_{\text {plim }}= & -\frac{\Lambda_{R}+i \Lambda_{I}}{\left(1-\cos \omega_{\mathcal{C}^{\prime}} T\right)+i \sin \omega_{\mathcal{C}^{\prime}} T} \\
=- & \frac{1}{2}\left[\frac{\Lambda_{R}\left(1-\cos \omega_{C^{\prime}} T\right)+\Lambda_{I} \sin \omega_{C^{\prime}} T}{1-\cos \omega_{C^{\prime}} T}+\right. \\
& \left.i \frac{\Lambda_{I}\left(1-\cos \omega_{C^{\prime}} T\right)-\Lambda_{R} \sin \omega_{C^{\prime}} T}{1-\cos \omega_{C^{\prime}} T}\right]
\end{aligned}
$$

$a_{\text {plim }}$ must be real, so equation (1.26) has only a real part and no imaginary part, giving $\Lambda_{I}\left(1-\cos \omega_{C^{\prime}} T\right)-$ $\Lambda_{R} \sin \omega_{C^{\prime}} T=0$. After simplifying by shifting the terms,

$$
\frac{\Lambda_{I}}{\Lambda_{R}}=\frac{\sin \omega_{C^{\prime}} T}{1-\cos \omega_{C^{\prime}} T}=\kappa
$$

$$
=\tan \Psi
$$

$\kappa$ is the number of leaflets of the stability wavefront map, taken as an integer. $\Psi$ is the phase shift of the eigenvalues; the dither-free axial depth of cut is obtained as

$$
a_{\text {plim }}
$$

$=-\frac{\Lambda_{R}}{2}\left(1+\kappa^{2}\right)$

Therefore, after determining $\omega_{C^{\prime}}$ and based on the expression (1.27), the relationship between the period $\mathrm{T}$ and the spindle speed $\mathrm{n}$, the relationship between the depth of cut and the speed of rotation, which represents the stability Lobe diagram, can be obtained as follows:

$$
\left\{\begin{array}{c}
\omega_{C^{\prime}} T=\pi-2 \Psi+2 k \pi \\
\mathrm{n}=\frac{1}{T}
\end{array}\right.
$$

1.2 Modal analysis of the disc cutter and cellular element wall plate

The 3D model is imported into the Abaqus simulation software, the disc cutter material properties are shown in Table 1, and the free meshing technique and C3D10 mesh cell are selected for meshing. The disc cutter is connected to the ultrasonic variable amplitude rod through the circular hole during the ultrasonic cutting process, and the deformation at the edge of the circular hole of the disc cutter is 0 . The solid support boundary condition is applied at the circular hole of the disc cutter. The initial analysis step and frequency extraction analysis step are set in the analysis step function module. The ultrasonic motion of the tool mainly vibrates in the form of longitudinal vibration, and the frequency modal analysis of the tool takes the first 30 orders of vibration and frequency of the tool. So, the demand eigenvalue parameter in the linear uptake analysis step is set 
Table 1 Disc cutter material parameters

\begin{tabular}{ccccc}
\hline Material & $\begin{array}{r}\text { Modulus of elasticity } \\
/ G p a\end{array}$ & $E_{C}$ & Poisson' s ratio & $\begin{array}{r}\text { Density } \\
\rho_{C} /(\mathrm{g} \\
\left./ \mathrm{cm}^{3}\right)\end{array}$ \\
\hline W6Mo5Cr4V2 & 200 & 0.3 & 7.8 \\
\hline
\end{tabular}

In this paper, the first 30 -order vibration patterns of the tool are extracted, in which the 5th-, 12th-, 17th- and 30th-order frequencies are different from their adjacent frequencies, which are $10738 \mathrm{~Hz}, 21904 \mathrm{~Hz}, 23386 \mathrm{~Hz}$ and 41454 , respectively, and their vibration patterns are shown in Fig. 3. The vibration displacement of the 5th- and 12th-order vibration patterns remains consistent at the same radius of the tool and increases monotonically along the radial direction of the tool, reaching the maximum amplitude at the cutting edge. The above vibration patterns meet the requirements of the maximum amplitude at the cutting edge of the ultrasonic tool, but the 5th-order vibration pattern also has axial bending. The 17th- and 30th-order amplitudes are more complicated and easily cause tool fracture. Meanwhile, the inherent frequency of the 12th order is consistent with the resonant characteristics of the operating frequency of the ultrasonic variable amplitude rod. In summary, the 12 th-order inherent frequency and vibration pattern meet the requirements of this paper.
The three-dimensional model of the cytoskeleton wall plate was established according to the actual dimensions, and its material properties are shown in Table 2. The modal analysis procedure is the same as that of the disc cutter, and the first-order inherent frequency of the cytoskeleton wall plate is $64517 \mathrm{~Hz}$, which is much larger than the 12th-order inherent frequency of the disc cutter.

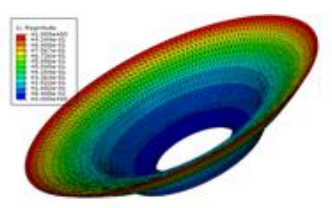

5th Power Vibration Type

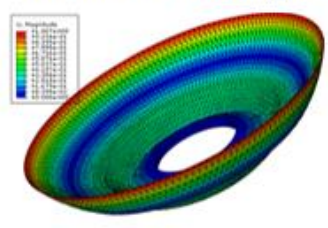

17th Power Vibration Type

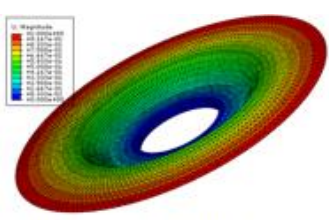

12th Power Vibration Type

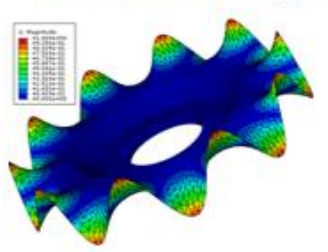

30th Power Vibration Type
Figure 3 5th-, 12th-, 17th- and 30th-order vibration patterns of the disc cutter

Table 2 Mechanical characteristics parameters of the cellular element wall plate of wave -absorbing honeycomb material

\begin{tabular}{|c|c|c|c|c|}
\hline Materials & $\begin{array}{c}\text { Modulus of } \\
\text { elasticity/(Mpa) }\end{array}$ & $\begin{array}{l}\text { Shear modulus } \\
\text { /(Мpa })\end{array}$ & $\begin{array}{c}\text { Poisson' }^{\prime}: \\
\text { ratio }\end{array}$ & $\begin{array}{l}\text { Density/ } \\
\left(\mathrm{kg} / \mathrm{m}^{3}\right)\end{array}$ \\
\hline cellular element wall plate & 544 & 3016 & 0.3 & 739.4 \\
\hline
\end{tabular}

\subsection{Cutting force coefficient analysis}

According to the mathematical stability model of the ultrasonic cutting process of the disc cutter established in a previous paper, it is known that to draw a complete stability Lobe diagram, in addition to obtaining the kinetic parameters of the disc cutter, it is also necessary to obtain the ultrasonic cutting force coefficient of the disc cutter. In the actual cutting process, the tool rotates with the spindle tangential cutting force $P_{t}$ and radial cutting force $P_{r}$ with the cutting depth of the tool $a_{p}$, and the related cutting force coefficient $\mathrm{K}$ and feed $f_{z}$ Error! Reference source not found.

$\left\{P_{t}=K_{t c} a_{p} f_{z}+K_{t e} a_{p}\right.$

$\left\{P_{r}=K_{r c} a_{p} f_{z}+K_{r e} a_{p}\right.$

where $K_{t c}, K_{r c}$ is the disc cutter $\mathrm{Z}$ direction, $\mathrm{X}$ is the cutting force coefficient in the direction $\left(\mathrm{N} / \mathrm{mm}^{2}\right)$ and $K_{t e}$ is $K_{r e}$ the $\mathrm{Z}$ direction $\mathrm{X}$ is the coefficient of cutting force in the direction $\left(\mathrm{N} / \mathrm{mm}^{2}\right)$ The coefficient is related to the edge wear of the tool. According to cutting experience, there is a certain degree of edge wear, and the edge is gradually rounded when the tool is cut.

\section{Experimental studies}

\subsection{Experimental bench construction}

Figure 5 shows the experimental setup for ultrasonic cutting of wave-absorbing honeycomb materials with a disc cutter, in which the self-researched ultrasonic cutting system has an operating frequency of $20 \mathrm{kHz}$, an output amplitude of $10-25 \mu \mathrm{m}$, and a power of $2 \mathrm{~kW}$ with an automatic frequency chasing function. The cutting force is collected by a Kistler force measuring sensor. 


\subsection{Cutting force coefficient calibration}

The ultrasonic amplitude was chosen to be $20 \mu \mathrm{m}$, and the ultrasonic frequency was $20 \mathrm{kHz}$. Disc cutting experiments with different cutting parameters were carried out to obtain the corresponding average cutting forces in the $\mathrm{X}$-direction and Z-direction, as shown in Table 3.

The obtained cutting forces were imported into MATLAB for fitting to obtain the cutting force coefficients and edge force coefficients for ultrasonic cutting of wave-absorbing honeycomb material by the disc cutter, as shown in Table 4.

Substituting the stability kinetic parameters in Table 5 into equation (1.20) for the relationship between ultrasonic cutting depth of cut and rotational speed to solve for the depth of cuta $\mathrm{p}_{\mathrm{p}}$ corresponding to the spindle speed $\mathrm{n}$ and using MATLAB programming in the set frequency range to plot the complete $n-a_{p}$ relationship plot, i.e., stability Lobe plot.
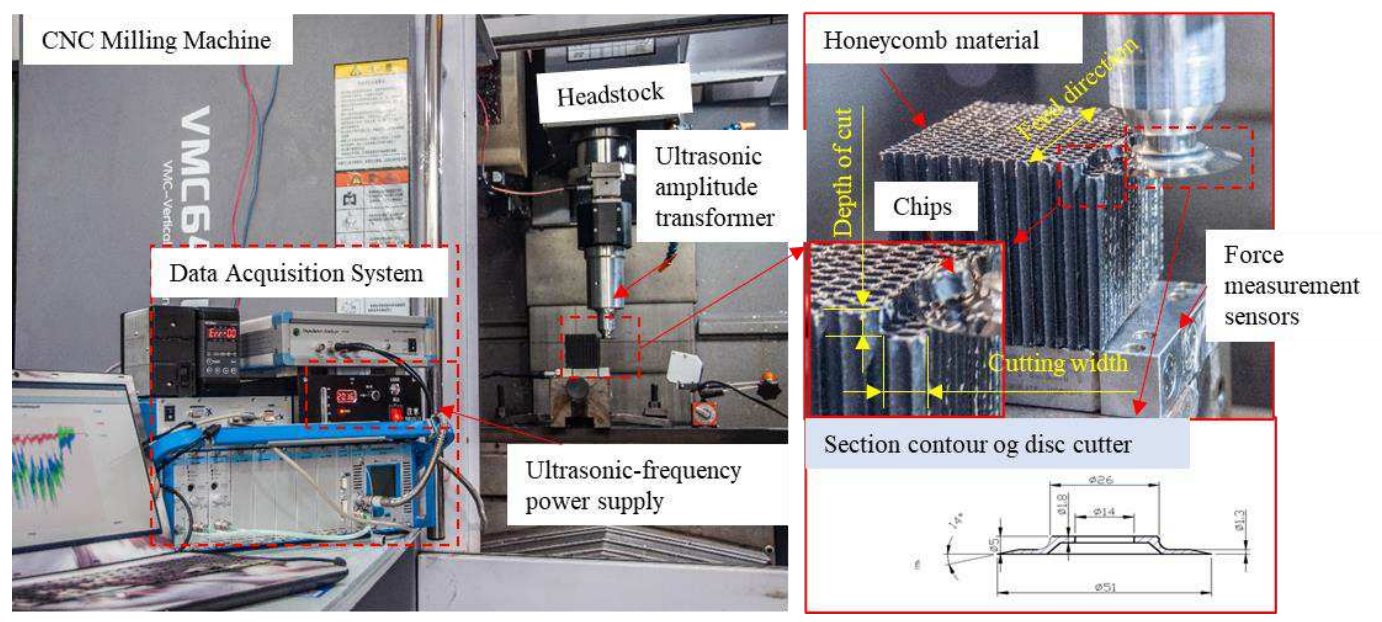

Fig. 4 Ultrasonic cutting experiment device for disc cutter

Table 3 Cutting forces obtained during cutting experiments with different cutting parameters

\begin{tabular}{ccccccc}
\hline number & $\begin{array}{c}\text { Feeding speed } \\
V_{f} /(\mathrm{m} / \mathrm{min})\end{array}$ & $\begin{array}{c}\text { Cutting deptr Feed amount } \\
a_{p} /(\mathrm{mm})\end{array}$ & $\begin{array}{c}\text { Tool speed } \\
f_{z} /(\mathrm{mm} / \mathrm{r})\end{array}$ & $\begin{array}{c}\text { Actual tangential } \\
n /(\mathrm{r} / \mathrm{min})\end{array}$ & $\begin{array}{c}\text { Actual radial } \\
\text { cutting force } P_{t} / \mathrm{N}\end{array}$ & \begin{tabular}{c} 
cutting force $P_{r} / \mathrm{N}$ \\
\hline 1
\end{tabular} \\
\hline 3 & 2 & 0.01 & 800 & 29.13 & 45.85 \\
2 & 3 & 2 & 0.02 & 800 & 30.52 & 46.37 \\
3 & 3 & 2 & 0.03 & 800 & 32.14 & 53.53 \\
4 & 5 & 2 & 0.03 & 1000 & 33.83 & 57.02 \\
5 & 5 & 4 & 0.03 & 1000 & 36.78 & 58.16 \\
6 & 5 & 2 & 0.02 & 1000 & 21.31 & 46.35 \\
7 & 10 & 3 & 0.02 & 1200 & 24.25 & 50.71 \\
8 & 10 & 4 & 0.02 & 1200 & 27.44 & 55.14 \\
\hline
\end{tabular}

Table 4 Cutting force coefficients and edge force coefficients for ultrasonic cutting of disc cutters

\begin{tabular}{ccccc}
\hline Coefficient & $K_{t c} /\left(\mathrm{N} / \mathrm{mm}^{2}\right)$ & $K_{r c} /\left(\mathrm{N} / \mathrm{mm}^{2}\right)$ & $K_{t e} /\left(\mathrm{N} / \mathrm{mm}^{2}\right)$ & $K_{r e} /\left(\mathrm{N} / \mathrm{mm}^{2}\right)$ \\
\hline Results & 75 & 192 & 14 & 21 \\
\hline
\end{tabular}

Table 5 Parameters required for plotting stability Lobe diagrams

\begin{tabular}{cccccccc}
\hline \multicolumn{2}{c}{ Disc cutter } & \multicolumn{2}{c}{ cellular element wall plate } & $K_{r c} /$ & $K_{t c} /$ & $\phi_{\text {in }}$ & $\phi_{\text {out }}$ \\
$\omega_{n}^{\prime} /(\mathrm{Hz})$ & $\xi_{n}^{\prime}$ & $\omega_{n}^{w}(\mathrm{~Hz})$ & $\xi_{n}^{w}$ & $\left(\mathrm{~N} / \mathrm{mm}^{2}\right)$ & $\left(\mathrm{N} / \mathrm{mm}^{2}\right)$ & $/\left(^{\circ}\right)$ & $/\left({ }^{\circ}\right)$ \\
\hline 21904 & 0.05 & 64517 & 0.05 & 192 & 75 & 0 & 90 \\
\hline
\end{tabular}




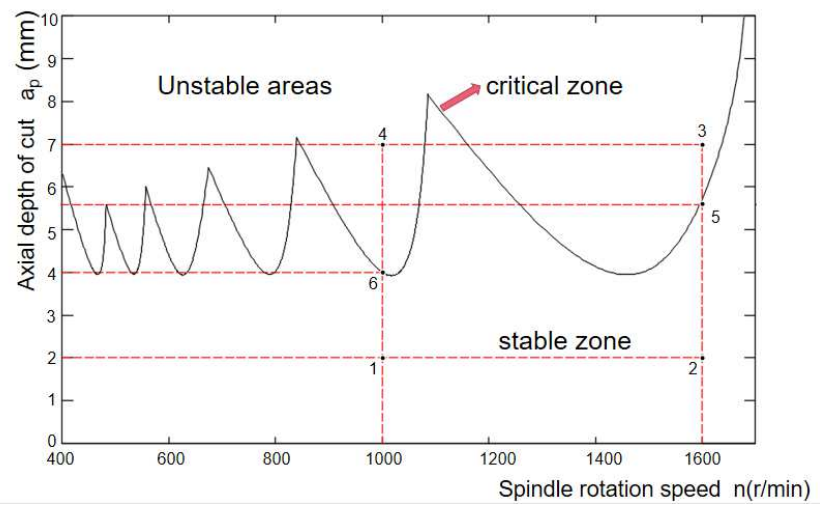

Figure 5 Lobe diagram of stability of ultrasonic cutting cytosolic siding by the disc cutter 
The results are shown in Figure 5.

As shown in Figure 5, when the axial depth of the cut was selected to be below $4 \mathrm{~mm}$, the tool was in a stable state when it was cut. When the axial cutting depth is selected from $4 \mathrm{~mm}-8 \mathrm{~mm}$, the tool is in a critical state of stability and instability when cutting, and then the tool needs to be selected at approximately $700 \mathrm{r} / \mathrm{min}, 900 \mathrm{r} / \mathrm{min}$ or $1200 \mathrm{r} / \mathrm{min}$ for stable cutting. When the cut depth is greater than $8 \mathrm{~mm}$, the cutting process is usually in a nonstable state. By looking at the whole leaf flap diagram, the right leaf flap is large and sparse, while the left leaf flap is small and dense. Therefore, to obtain stable machining process parameters for a larger cut depth, it is necessary to find parameters in a higher speed range.

\subsection{Ultrasonic disc cutter cutting stability}

\section{experiments and results analysis}

To verify the accuracy of the stability Lobe diagram, the tool speed and depth of cut were selected in the stable region, unstable region and critical stable region shown in Figure 5 to carry out ultrasonic cutting experiments with the disc cutter, as shown in points $1-6$ within Figure 5, and the specific values are shown in Table 6 .

Table 6 Experimental parameters for stability Lobe plot accuracy verification

\begin{tabular}{ccccc}
\hline Region Serial number & $\begin{array}{c}\text { Cutting } \\
\text { depth } a_{p} / \mathrm{mm}\end{array}$ & $\begin{array}{c}\text { Tool speed } \\
\mathrm{n} / \mathrm{mm}\end{array}$ & $\begin{array}{c}\text { Feeding speed } V_{f} \\
/(\mathrm{m} / \mathrm{min})\end{array}$ \\
\hline Stable area & 1 & 2 & 1000 & 5 \\
Unstable area & 3 & 2 & 1600 & 5 \\
Critical stability & 5 & 5.5 & 1600 & 5 \\
area & 4 & 4 & 1000 & 5 \\
\hline
\end{tabular}

Figures 6a) and 6b) show the machined surfaces after cutting with the process parameters at points 1 and 2 in the stable area of the Lobe diagram, respectively. The surface of the workpiece is neat and smooth, the fibre layer fracture is flat, only a small number of positions have a slight fibre layer pulling fracture, the resin layer does not produce peeling, and only a small number of local fractures occur. Figure 6c) and 6d) show the machined surfaces after cutting with process parameters of 5 and 6 points in the critical area of the lobe diagram, respectively. There is a clear deformation of the fibre layer in the cell wall area and a large amount of separation of the resin layer from the fibre layer in the cell cross area. Figures 6e) and 6f) show the machined surfaces after cutting with process parameters of 3 and 4 points in the unstable region of the Lobe diagram, respectively. It can be observed that the cell wall area shows severe fibre layer pulling, deformation and fracture, and the cell cross area shows the separation of the resin layer and fibre layer on a larger scale. The analysis also shows that the damage of the fibre layer mainly occurs at the cell wall, while the damage of the resin layer, such as fracture and separation, mainly occurs at the intercellular intersection. According to structural mechanics, these two locations show different mechanical properties due to their different structures, with the cell wall showing higher elasticity and plasticity and the cell intersection showing higher strength. Since the resin material has a weaker tensile capacity and more obvious brittleness compared with the fibre material, it can be introduced that the cutting force is greater in the Lobe diagram instability region and the critical region when selecting process parameters for processing, which leads to large scale deformation of the cytomel wall, damage characteristics are mainly manifested as deformation, drawing and fracture of the fibre layer, while the material deformation is smaller in the cytomel intersection region, so the damage characteristics The damage is mainly characterized by the fracture and peeling of the resin layer. 


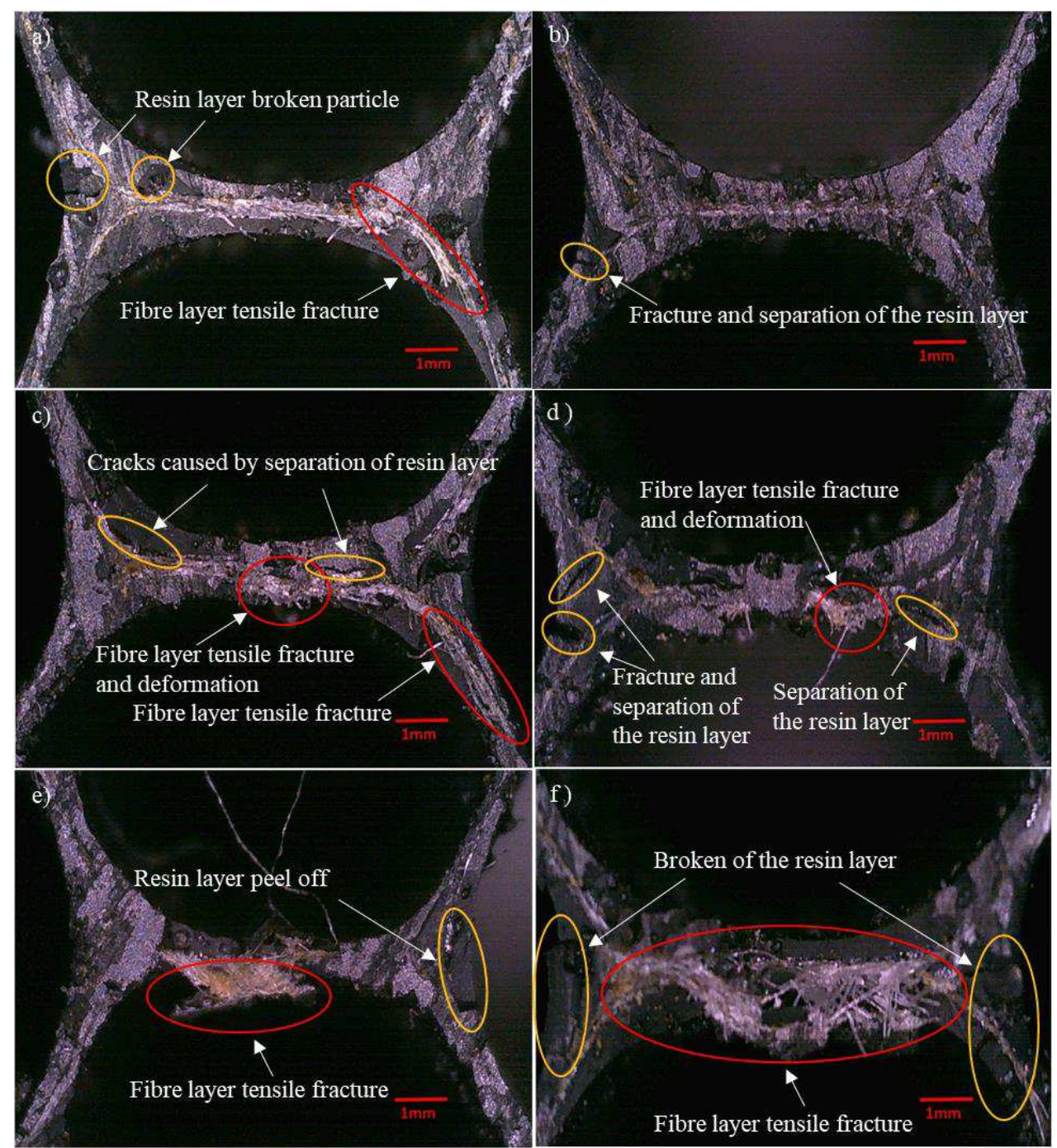

Figure 6 Microscopic surface profile of the wave-absorbing honeycomb material after ultrasonic cutting with a disc cutter

Figure 7 shows the changes in the number of notches and notch pattern of the disc cutter edge with cutting distance during ultrasonic cutting using the process parameters represented by points 1, 4 and 6 in Figure 5. It can be seen from the figure that when the cutting parameters of unstable area and critical area are selected for machining, the disc cutter edge is broken when the cutting distance reaches 300 $\mathrm{mm}$, and the edge breakage increases rapidly with the increase of cutting distance. For disc cutter cutting in unstable conditions, the number of edge notches increases rapidly, and the notch increases significantly after the cutting distance reaches $500 \mathrm{~mm}$. For disc cutter cutting in the critical state, when the cutting distance reaches $1000 \mathrm{~mm}$, the number of cutting-edge notches increases rapidly, the notch depth also increases significantly, and there is high-temperature smoke and burn. When the stable area cutting parameters were selected for machining, edge breakage was found only when the cutting distance reached $800 \mathrm{~mm}$; when the cutting distance reached $1500 \mathrm{~mm}$, a large number of edge notches appeared; if machining continued, large notches appeared on the edge, the machining process gradually destabilized, high temperature burn marks appeared on the tool surface, and the machined surface deteriorated sharply. By comparing the cutting edge notch patterns generated under different process parameters, it can be found that the cutting edge notches generated by machining with non-stable zone and critical zone cutting parameters are discrete thin strips of broken off, while the cutting edge notches generated by machining with stable zone are semi-circular broken and usually accumulate. As the machining continues, the notches will continue to increase together leading to the accumulation of poor machining condition. 


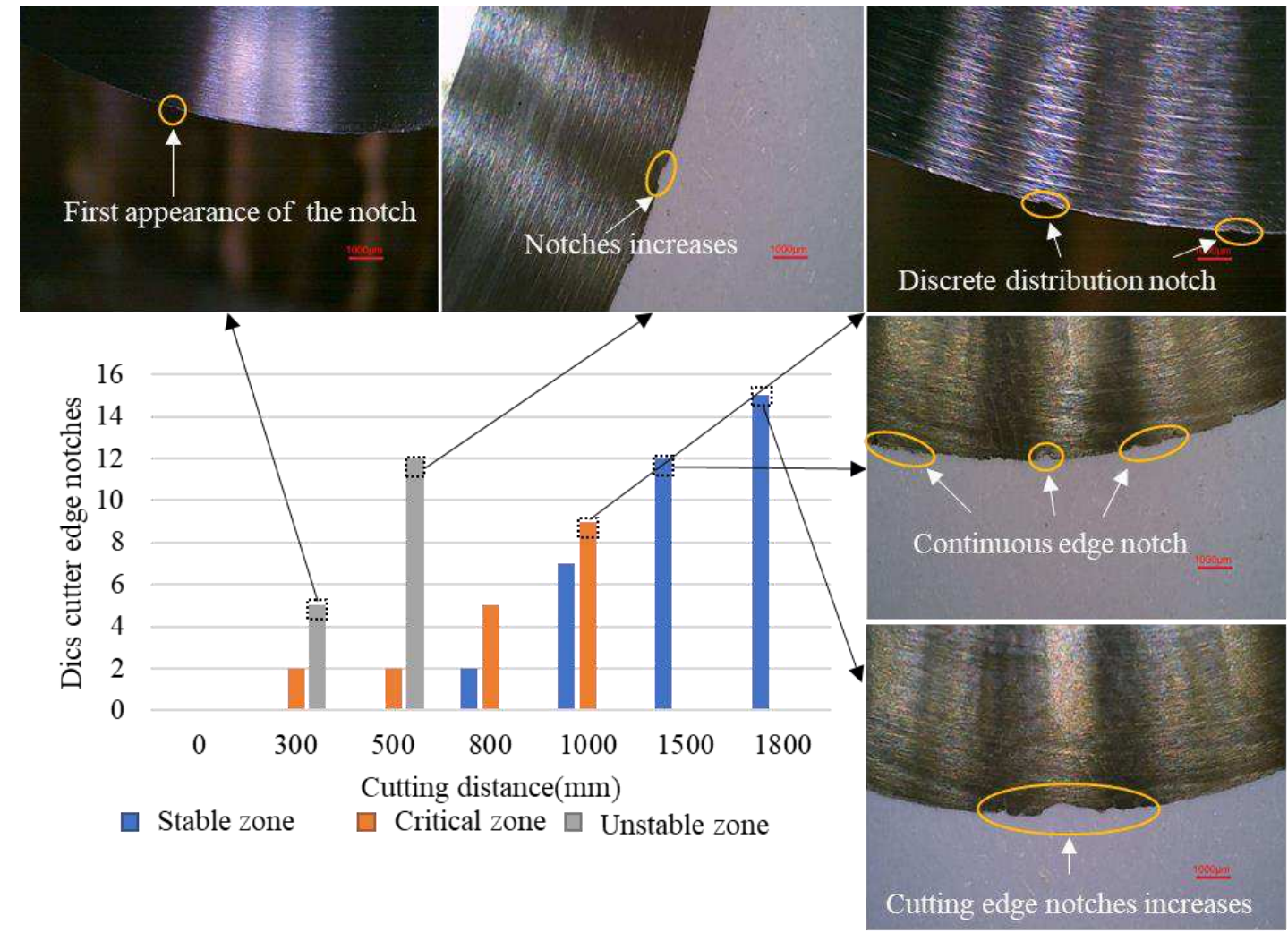

Fig. 7 Variation in the number of broken points of the disc cutter

The above experimental results show that selecting cutting parameters in the stable region of the Lobe diagram for machining, the machined surface has a better finish and the machining quality is much higher than the cutting parameters selected in the critical stable region and unstable region. Selecting process parameters in the stable region of the Lobe diagram for machining can significantly improve the tool life, which proves that the theoretical model and stability established in the Lobe diagram in this paper can be used to guide the cutting processing of honeycomb materials.

\section{Conclusion}

In this paper, by establishing a mathematical model of an ultrasonic disc cutter and honeycomb cellular element wall plate, using MATLAB to draw the stability Lobe diagram of the ultrasonic cutting tool, and building the ultrasonic cutting test bench of the disc cutter, ultrasonic cutting experiments were conducted on wave-absorbing honeycombs at different rotational speeds and axial cutting depths. By observing the surface of the honeycomb after cutting and the broken state of the tool, the optimal depth of cut and spindle speed during ultrasonic cutting were analysed in combination with the stability Lobe diagram. By comparison, the following conclusions can be drawn:

(1) The experimental results are consistent with the overall trend predicted by the stability Lobe diagram, which verifies the correctness of the mathematical model of the multi-degree-of-freedom stability of the ultrasonic cutting system.
(2) For the ultrasonic disc tool with a diameter of 50 $\mathrm{mm}$ in ultrasonic cutting, the axial depth of cut is selected from $1 \mathrm{~mm}$ to $4 \mathrm{~mm}$, and the spindle speed is selected from $800 \mathrm{r} / \mathrm{min}$ to $1200 \mathrm{r} / \mathrm{min}$ to obtain the best quality of honeycomb cutting surface. These selections lay a solid foundation for the selection of wave-absorbing honeycomb processing process parameters.

(3) Due to the different structural strengths of the cytosolic wall and the cytosolic intersection, as well as the different mechanical properties of the resin layer material and the fibre layer material, damage to the fibre layer mainly occurs at the location of the cytosolic wall, while damage such as fracture and separation of the resin layer mainly occurs at the location of the cytosolic intersection.

(4) Compared with the unstable zone and the critical zone, the tool life is significantly improved by selecting the stable zone process parameters for cutting. By comparing the cutting-edge notch morphology, it can be found that the discrete, strip-shaped notches produced by selecting the unstable and critical zone process parameters are closer to the form of fatigue breakage, while the continuous, semi-circular notches produced by selecting the stable zone process parameters are closer to the form of breakage under normal operation.

Author contribution: Xiaoping $\mathrm{Hu}$, Baohua $\mathrm{Yu}$ and Hongxian Ye planned the research. Xin Liu and Sufang Yao did experiments. Zongfu Guo wrote the paper and 
analyzed the data.

Funding: The National Natural Science Foundation of China (No.51975173), Zhejiang Province Public Welfare Technology Application Research Project (No.

LGG21E050010), Fundamental Research Funds for the Provincial Universities of Zhejiang (No. GK199900X018)

Data availability: This article contains all the data gathered or analyzed during this study.

Ethical approval: None of the studies mentioned in this article contain any human participation. Also, no animals were harmed during these experiments.

Consent to participate: The authors consent to participate.

Consent for publication: The authors provide their consent to publish this article.

Competing interests: The authors declare no competing interests.

\section{References}

[1] Hu Sheng. Application of Genetic Algorithm in Honeycomb Structured Wave Absorbing Materials[D]. Huazhong University of Science and Technology,2019. [2] Mohamed Jaafar, Mohammed Nouari, Hamid Makich, Abdelhadi Moufki. 3D numerical modeling and experimental validation of machining Nomex ${ }^{\circledR}$ honeycomb materials $[\mathrm{J}]$. The International Journal of Advanced Manufacturing Technology,2021(prepublish):

[3] Ahmad Shahzad et al. Experimental study on rotary ultrasonic machining (RUM) characteristics of Nomex honeycomb composites (NHCs) by circular knife cutting tools[J]. Journal of Manufacturing Processes, 2020, 58 : 524-535.

[4] Jiaming Jiang, Zhanqiang Liu. Formation mechanism of tearing defects in machining Nomex honeycomb core $[\mathrm{J}]$. The International Journal of Advanced Manufacturing Technology,2021(prepublish):

[5] U. Bravo et al. Stability limits of milling considering the flexibility of the workpiece and the machine[J]. International Journal of Machine Tools and Manufacture, 2005, 45(15): 1669-1680.

[6] V. Thevenot et al. Influence of Material Removal on The Dynamic Behavior of Thin-Walled Structures in Peripheral
Milling[J]. Machining Science and Technology, 2006, 10(3): 275-287.

[7] X-M Zhang and L-M Zhu and H Ding. Matrix perturbation method for predicting dynamic modal shapes of the workpiece in high-speed machining[J]. Proceedings of the Institution of Mechanical Engineers, Part B: Journal of Engineering Manufacture, 2010, 224(1): 177-183.

[8] Iker Mancisidor et al. Receptance coupling for tool point dynamic prediction by fixed boundaries approach[J]. International Journal of Machine Tools and Manufacture, 2014, 78: 18-29.

[9] Zhang Xuewei, Yu Tianbiao, Wang Wanshan. Modeling and analysis of three-dimensional chattering stability of thin-walled parts milling[J]. Journal of Northeastern University (Natural Science Edition),2015,36(01):99-103. [10] Zhu Hui. Study on the chatter stability of longitudinal-torsional composite ultrasonic vibration milling of thin plate parts[D]. Harbin Institute of Technology,2018. [11] Wu, Chen-Jun. Development of longitudinal torsional resonance ultrasonic milling system and its machining experimental research[D]. Harbin Institute of Technology,2018. 